\title{
Time-dependent variations in milk fatty acid content of goats fed 3 different plant oils
}

\author{
A. L. Martínez Marín, ${ }^{*}$ P. Gómez-Cortés, $\dagger^{1}$ G. Gómez Castro, ${ }^{*} M$. Juárez, $†$ L. Pérez Alba, ${ }^{*}$ \\ M. Pérez Hernández, ${ }^{*}$ and M. A. de la Fuente ${ }^{2}$ \\ *Departamento de Producción Animal (Universidad de Córdoba), Ctra. Madrid-Cádiz s/n, Campus de Rabanales, 14014 Córdoba, Spain \\ †Instituto de Investigación en Ciencias de la Alimentación (CSIC-UAM), Nicolás Cabrera, 9. Universidad Autónoma de Madrid, 28049 Madrid, \\ Spain
}

\section{ABSTRACT}

The effect of sampling time on milk fatty acid (FA) composition after separately adding 3 plant oils to an oil-free control diet (67\% cereal-soybean-based concentrate and $33 \%$ alfalfa hay) was studied in 12 Malagueña goats. Individual animals were randomly allocated to 1 of the 4 treatments: control, $48 \mathrm{~g} / \mathrm{d}$ of added high oleic (OSO) or regular (RSO) sunflower oil, or linseed oil (LO). Individual milk samples were taken at 0 (covariate), 1, 12, 24, 72, 120, 192, 312, and $504 \mathrm{~h}$ after the beginning of the experiment. Milk FA contents (g/100 g of total FA methyl esters) were analyzed in a completely randomized design with repeated measures using PROC MIXED of SAS (SAS Institute Inc., Cary, NC). Comparing results of 15 chosen FA (for example, medium-chain saturated FA trans-11 C18:1, cis-9,trans-11 $\mathrm{C} 18: 2$, trans-10 $\mathrm{C} 18: 1$, and $\mathrm{C} 18: 3 \mathrm{n}-3)$ indicated that throughout the duration of the experiment, feeding the control diet had little influence on the concentrations of most FA in milk. Most changes in milk FA composition due to oil supplementation had occurred within $192 \mathrm{~h}$ since the beginning of the experiment. However, the concentrations of 2 FA (trans-10 C18:1 in RSO and $\mathrm{C} 18: 3 \mathrm{n}-3$ in LO treatments) continued to change until $504 \mathrm{~h}$. By comparing FA values in milk fat from oil treatments with those of the control at the same sampling times, typical value differences for the 3 supplementary oils found at $504 \mathrm{~h}(21 \mathrm{~d})$ were also observed at $312 \mathrm{~h}$ from the beginning of the experiment $(13 \mathrm{~d})$ and even earlier in some FA, such as mediumchain saturated FA at $120 \mathrm{~h}$ in RSO and LO and at 72 $\mathrm{h}$ in OSO, cis-9,trans-11 C18:2 and trans-10 C18:1 at $24 \mathrm{~h}$ in RSO, trans-11 C18:1 at $12 \mathrm{~h}$ in RSO and LO, and $\mathrm{C} 18: 3 \mathrm{n}-3$ at $1 \mathrm{~h}$ in LO. In the conditions assayed in

Received October 26, 2012.

Accepted January 24, 2013.

${ }^{1}$ Present address: Division of Nutritional Sciences, Cornell University, Ithaca, NY 14853.

${ }^{2}$ Corresponding author: mafl@if.csic.es these experiments, reliable results of milk FA changes were obtained at sampling times shorter than $21 \mathrm{~d}$. Monitoring early changes in milk FA after the addition of plant oils to diets could help in the study of rumen and mammary metabolism of dietary FA.

Key words: plant oil, fatty acid, goat milk, sampling time

\section{INTRODUCTION}

There is a scarcity of information dealing with the kinetics of milk FA composition responses to plant oil inclusions in the diet of dairy ruminants. Most information corresponds to work carried out with cows (Dhiman et al., 2000; Roy et al., 2006) and ewes (Gómez-Cortés et al., 2008a,b; Hervás et al., 2008). To our knowledge, only Chilliard et al. (2005) gave data on time-related changes to cis-9, trans-11 C18:2 content after introducing sunflower or linseed oils into dairy goat diets.

In most published papers, the first milk sample was taken 2 or $7 \mathrm{~d}$ after the introduction of the plant oil into the diets. However, in vitro work done by Mosley et al. (2002) and Jouany et al. (2007) showed changes in the accumulation of biohydrogenation (BH) intermediates and end products as soon as $0.5 \mathrm{~h}$ after oil inclusion in media inoculated with mixed rumen microbes. Fievez et al. (2007) indicated that rumen microorganisms are permanently adapted to biohydrogenate unsaturated FA from plants because they are always in contact with them in most ruminant diets. Different authors (Moate et al., 2004; Harvatine and Allen, 2006) have used information from in vivo experiments to build models describing rumen $\mathrm{BH}$ kinetics. These models predict a fast, strong lipolysis-BH reaction, which delivers unsaturated fats into the rumen and provide estimates for FA rumen passage rates. Whereas the model by Moate et al. (2004) practically precludes C18:3n-3 from escaping the rumen unaltered by $\mathrm{BH}$, the model by Harvatine and Allen (2006) allows room for this to happen.

When studying the kinetics of FA responses to the inclusion of plant oils in dairy ruminant diets, one has 
to bear in mind that the observed effects on milk FA changes also imply the time from FA leaving the rumen to their secretion as milk triacylglycerols. Furthermore, although several authors have studied the response of milk FA composition to abomasal/duodenal infusions of long-chain FA (Drackley et al., 2007; Khas-Erdene et al., 2010), their studies show changes occurring in the milk fat FA profile at $5 \mathrm{~d}$ from the beginning of the infusions, at the earliest. The aim of this work was to obtain information about the timing of changes in FA contents in goat milk fat from 1 to $504 \mathrm{~h}(21 \mathrm{~d})$ after introducing 3 different plant oils into the diet.

\section{MATERIALS AND METHODS}

The experiments were carried out on the premises of the Animal Production Department of Córdoba University (Córdoba, Spain). Animals were kept in accordance with Spanish regulations relative to the treatment of experimental animals. Twelve Malagueña goats were used ( $45 \pm 5$ DIM, $47 \pm 4.2 \mathrm{~kg}$ live weight, and 2,287 \pm $512 \mathrm{~g}$ of milk production/d at the beginning of the ex- periment). They were placed in individual cages of 1.0 $\times 1.4 \mathrm{~m}$ with slatted floors equipped with water and feeding troughs. All goats were fed a general-purpose diet without added fat (maize, oats, horsebeans, and alfalfa hay) from kidding until the beginning of the experiment.

Goats were randomly assigned to 1 of 4 treatments (3 goats per treatment): a basal control diet (67\% concentrate and $33 \%$ alfalfa hay) with no added oil or a basal diet supplemented with $48 \mathrm{~g} / \mathrm{d}$ of either high oleic sunflower oil (OSO), regular sunflower oil (RSO), or linseed oil (LO) as shown in Table 1. The experiments lasted $21 \mathrm{~d}$. Milk samples were taken from milkings at $0 \mathrm{~h}$ (before oil supplementation) and 1, 12, 24, 72, 120, 192,312 , and $504 \mathrm{~h}$ after the addition of the corresponding oil. Milkings at 0 (covariate), 1 , and $12 \mathrm{~h}$ were stripped out by hand after giving an i.v. dose of 2 to 3 IU of oxytocin to the goats. Daily DMI, BW changes, milk production and sampling, and diet analysis were carried out as in Martínez Marín et al. (2012).

Milk fats were extracted as described by Gómez-Cortés et al. (2008a). Fatty acid methyl esters (FAME)

Table 1. Ingredients, chemical composition, and nutritive value of the experimental diets

\begin{tabular}{|c|c|c|c|c|}
\hline \multirow[b]{2}{*}{ Item } & \multicolumn{4}{|c|}{ Treatment $^{1}$} \\
\hline & Control $^{2}$ & OSO & $\mathrm{RSO}$ & LO \\
\hline \multicolumn{5}{|l|}{$\operatorname{Diet}(\mathrm{g} / \mathrm{d})$} \\
\hline Alfalfa hay & 600 & 600 & 600 & 600 \\
\hline Concentrate $^{3}$ & 1,200 & 1,200 & 1,200 & 1,200 \\
\hline $\mathrm{OSO}^{4}$ & - & 48 & - & - \\
\hline $\mathrm{RSO}^{4}$ & - & - & 48 & - \\
\hline $\mathrm{LO}^{4}$ & - & - & - & 48 \\
\hline \multicolumn{5}{|l|}{ Chemical composition } \\
\hline DM $(\%)$ & 90.6 & 90.5 & 91.0 & 91.2 \\
\hline $\mathrm{CP}(\%$ of DM) & 17.0 & 16.4 & 16.4 & 16.5 \\
\hline NDF (\% of DM) & 28.2 & 27.5 & 27.0 & 26.9 \\
\hline $\operatorname{AHEE}^{5}(\%$ of DM) & 2.4 & 5.6 & 5.5 & 5.8 \\
\hline \multirow{2}{*}{\multicolumn{5}{|c|}{ Nutritive value ${ }^{6}$}} \\
\hline & & & & \\
\hline $\mathrm{ME}(\mathrm{Mcal} / \mathrm{kg}$ of $\mathrm{DM})$ & 2.67 & 2.77 & 2.77 & 2.77 \\
\hline $\mathrm{MP}(\mathrm{g} / \mathrm{kg}$ of $\mathrm{DM})$ & 123 & 119 & 119 & 119 \\
\hline \multicolumn{5}{|l|}{ FA from oil $(\mathrm{g} / \mathrm{d})$} \\
\hline C16:0 & - & 1.8 & 2.9 & 2.6 \\
\hline C18:0 & - & 1.4 & 2.0 & 1.8 \\
\hline cis-9 C18:1 & - & 41.0 & 14.2 & 10.0 \\
\hline $\mathrm{C} 18: 2 \mathrm{n}-6$ & - & 2.7 & 27.9 & 8.0 \\
\hline C18:3n-3 & - & - & - & 23.9 \\
\hline
\end{tabular}

${ }^{1}$ Control = basal diet with no added oil; OSO, RSO, and LO = diets enriched with $48 \mathrm{~g} / \mathrm{d}$ of high-oleic sunflower oil, regular sunflower oil, and linseed oil, respectively.

${ }^{2}$ Control diet supplied 5.1, 0.8, 6.9, 18.9, and $6.5 \mathrm{~g} / \mathrm{d}$ of C16:0, C18:0, cis-9 C18:1, cis-9,cis-12 C18:2, and C18:3n-3, respectively; calculated according to INRA (2002).

${ }^{3}$ Composition (g/kg, as fed): maize, 375; barley, 374.9; soybean meal, 200; vitamin and mineral premix (Maxi Nutral Ovejas; Nutral SA, Madrid, Spain), 30; binder (Exal; Tolsa S.A., Madrid, Spain), 20; antioxidant (Luctanox; Lucta S.A., Barcelona, Spain), 0.1.

${ }^{4}$ Included in the respective concentrates. High-oleic sunflower oil and RSO were purchased from Carrefour S.A. (Madrid, Spain). Linseed oil was supplied by Gustav Heess S. L. (Barcelona, Spain).

${ }^{5}$ Acid hydrolysis ether extract.

${ }^{6}$ Calculated from NRC (2007). 

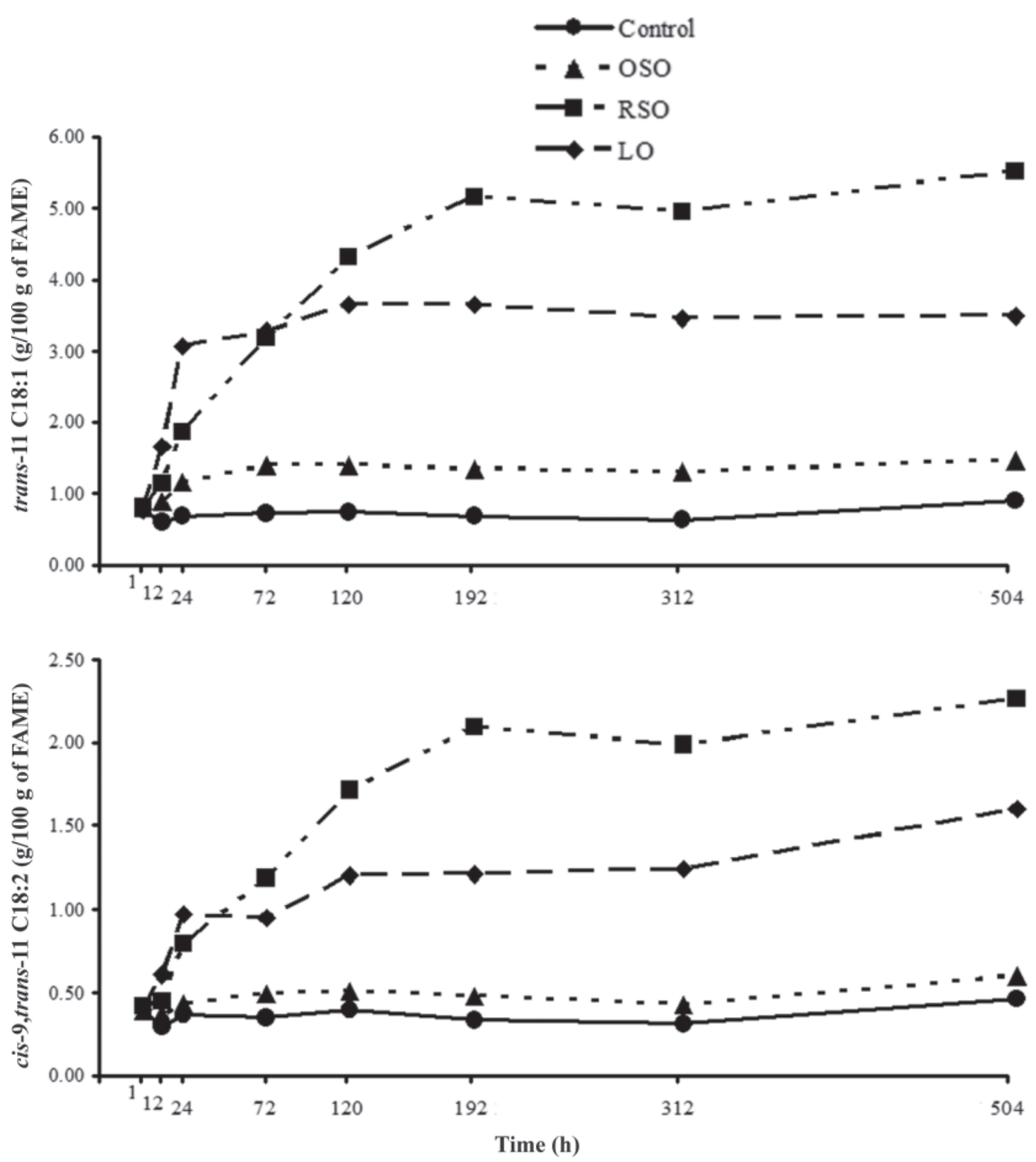

Figure 1. Time-dependent changes of trans-11 C18:1 and cis-9,trans-11 C18:2 in milk fat of goats fed an unsupplemented diet (control) or diets enriched with high oleic sunflower oil (OSO), regular sunflower oil (RSO), or linseed oil (LO). FAME = FA methyl esters.

were prepared by base-catalyzed methanolysis of the glycerides (ISO-IDF, 2000a). Analysis of FAME was performed on a gas-liquid chromatograph (Agilent 6890 N Network System; Agilent Technologies Inc., Santa Clara, CA) onto a CP-Sil 88 fused silica capillary column $(100 \mathrm{~m} \times 0.25 \mathrm{~mm}$; Varian BV, Middelburg, the Netherlands) under similar conditions to those reported by Luna et al. (2008). Individual FAME quantification was performed according to ISO-IDF (2002b) using a milk fat with known composition (CRM 164; European Community Bureau of Reference, Brussels, Belgium). Individual FA were identified by comparison with stan- dards distributed by Nu-Chek Prep Inc. (Elysian, MN), whereas trans-11,cis-15 C18:2, trans-11,trans-15 C18:2, cis-9,trans-11,cis-15 C18:3, and cis-9,trans-11,trans-15 C18:3 had previously been identified by gas chromatography-tandem mass spectrometry (Gómez-Cortés et al., 2009).

Data of milk fat FA content were analyzed by repeated measurement analysis (Littell et al., 2006) using PROC MIXED of SAS (SAS Institute, 2004). The statistical model included the fixed effects of diet, time, their interaction, the covariate, and the random effect of goat nested within treatment. For each FA, the 
covariance structure of goat nested within treatment [compound symmetry, $\operatorname{ANTE}(1)$ or spatial power] was chosen on the basis of the Schwarz Bayesian information model fit criteria. Least squares means (adjusted by the covariate) of the diet $\times$ time interaction were compared using the CONTRAST statement in PROC MIXED. Within treatments, the least squares means were compared with their respective 504-h values. Least squares means from samples of goats fed with added oil were also compared, by sampling times, with the respective control mean. Statistical differences were declared at $P<0.05$.

\section{RESULTS AND DISCUSSION}

To study the effect of time after introducing the oils into the diets, $15 \mathrm{FA}$ were selected (Table 2) on the basis of their significant differences in diet $\times$ time interaction and relevance in current knowledge of rumen and mammary metabolism as well as in human nutrition.

\section{Comparing Respective 504-h Values}

In the control treatment, sampling time did not affect the proportion of any FA in Table 2 [i.e., for a given $\mathrm{FA}$, the values obtained at any sampling time were no different from the corresponding 504-h value $(21 \mathrm{~d})$ ]. This provided a proper reference for comparing the effects of sampling time on the 3 oil treatments.

In the OSO treatment, only $4 \mathrm{FA}$ out of the 15 presented in Table 2 showed differences due to sampling time compared with the corresponding value obtained at 504 h: medium-chain SFA (MCSFA), C18:0, cis-9 C18:1, and trans-15(+cis-11) C18:1. No significant differences were observed in the percentages of MCSFA and C18:0 relative to the final samples collected after 1 and $3 \mathrm{~d}$, respectively. The effect of OSO on MCSFA content in milk fat may be mediated by 2 different causes, which are not mutually exclusive: one is the negative effect of oil on VFA produced in rumen fermentation, and the other is the direct effect of long-chain FA absorbed in the small intestine on de novo FA synthesis in the mammary gland. According to Martínez Marín et al. (2012), who used the same diets in their experiments as those in the current work, the likelier cause is the second because the short-chain SFA (SCSFA; C4:0 to C8:0) showed no negative, or even positive, responses to oil supplementation (Table 2). The effect of OSO on C18:0 and cis-9 C18:1 milk fat content probably reflected its high level of cis-9 C18:1 and the particular rumen BH-mammary desaturation cycle of these FA.

Five FA in the RSO treatment showed values statistically different from their corresponding 504-h values, which did not differ significantly at $72 \mathrm{~h}$ (MCSFA and cis-9,cis-12 C18:2), at $120 \mathrm{~h}$ (trans-11 $\mathrm{C} 18: 1$ and cis9,trans-11 C18:2; Figure 1) or at 192 h (SCSFA; i.e., between 3 and 8 d; Table 2). Roy et al. (2006) and Shingfield et al. (2006) observed in cows and GómezCortés et al. (2008a, 2011) in ewes, that the contents of trans-11 C18:1 and cis-9,trans-11 C18:2 in milk fat reached maximum values between 6 and $7 \mathrm{~d}$ after introducing linoleic acid-rich oils into the diet, but then decreased and leveled off to values higher than those of the control diet. In the current research, the highest milk fat content values of these $2 \mathrm{FA}$ were reached at $8 \mathrm{~d}$, but rather than subsequently decreasing, they leveled off at these highest values. On the other hand, the sampling time values of trans-10 C18:1 were all different from the corresponding 504-h value, which was higher than the apparent plateau reached between 120 and 312 $\mathrm{h}$ (Figure 2). This type of response was not observed in cows [Roy et al. (2006), with forage:concentrate ratios of 27:73 and 48:52; or by Shingfield et al. (2006), with a forage:concentrate ratio of $60: 40]$. Both sets of authors reported a plateau of trans-10 C18:1 content in milk fat around 10 to $12 \mathrm{~d}$, up to 20 and $28 \mathrm{~d}$, respectively. However, Gómez-Cortés et al. (2008a) found the highest value of trans-10 C18:1 on the last sampling day of their experiment $(21 \mathrm{~d})$ with a 20:80 forage:concentrate diet supplemented with $6 \%$ of soybean oil. GómezCortés et al. (2011) also found the same type of result at $28 \mathrm{~d}$ when including $2 \%$ of sunflower oil in a $30: 70$ forage:concentrate diet. All of this suggests that temporal changes in trans-10 C18:1 milk fat content observed in cows could be different from those in ewes and goats. In these species, at least with high-concentrate diets, the potential maximum value of trans-10 $\mathrm{C} 18: 1$ content in milk fat does not seem to have been reached at 21 or $28 \mathrm{~d}$ after introducing the dietary oil.

Nine of the 15 FA listed in Table 2 that showed statistically significant differences between their first sampling time values and those of the corresponding 504-h sampling in the LO treatment did not differ significantly at $24 \mathrm{~h}$ [MCSFA, trans-11 C18:1, trans15(+cis-11) C18:1, cis-9,cis-12 C18:2, cis-9,trans-11 C18:2, and cis-9,trans-11,cis-15 C18:3], at $120 \mathrm{~h}$ (cis$15 \mathrm{C} 18: 1$ ), or at $192 \mathrm{~h}$ (trans-11,cis-15 C18:2 and cis9,trans-11,trans-15 C18:3). The content of C18:3n-3 showed numerically increasing values with time, which were all lower than the 504-h value (Table 2), suggesting that the potential maximum content of $\mathrm{C} 18: 3 \mathrm{n}-3$ in milk fat was not reached before or even at $504 \mathrm{~h}(21$ d) after the introduction of LO in the dairy goat diets (Figure 2). A similar response was observed by Luna et al. (2005) working with ewes fed linseed for $6 \mathrm{wk}$.

In the current study, the final values of trans-11 C18:1 and cis-9,trans-11 C18:2 were 58 and $42 \%$ higher, respectively, in the RSO than in the LO treatment. 
Table 2. Fatty acid content (g/100 g of FA methyl esters) at different sampling times in milk fat from goats fed control (basal diet with no added oil) or oil-supplemented diets

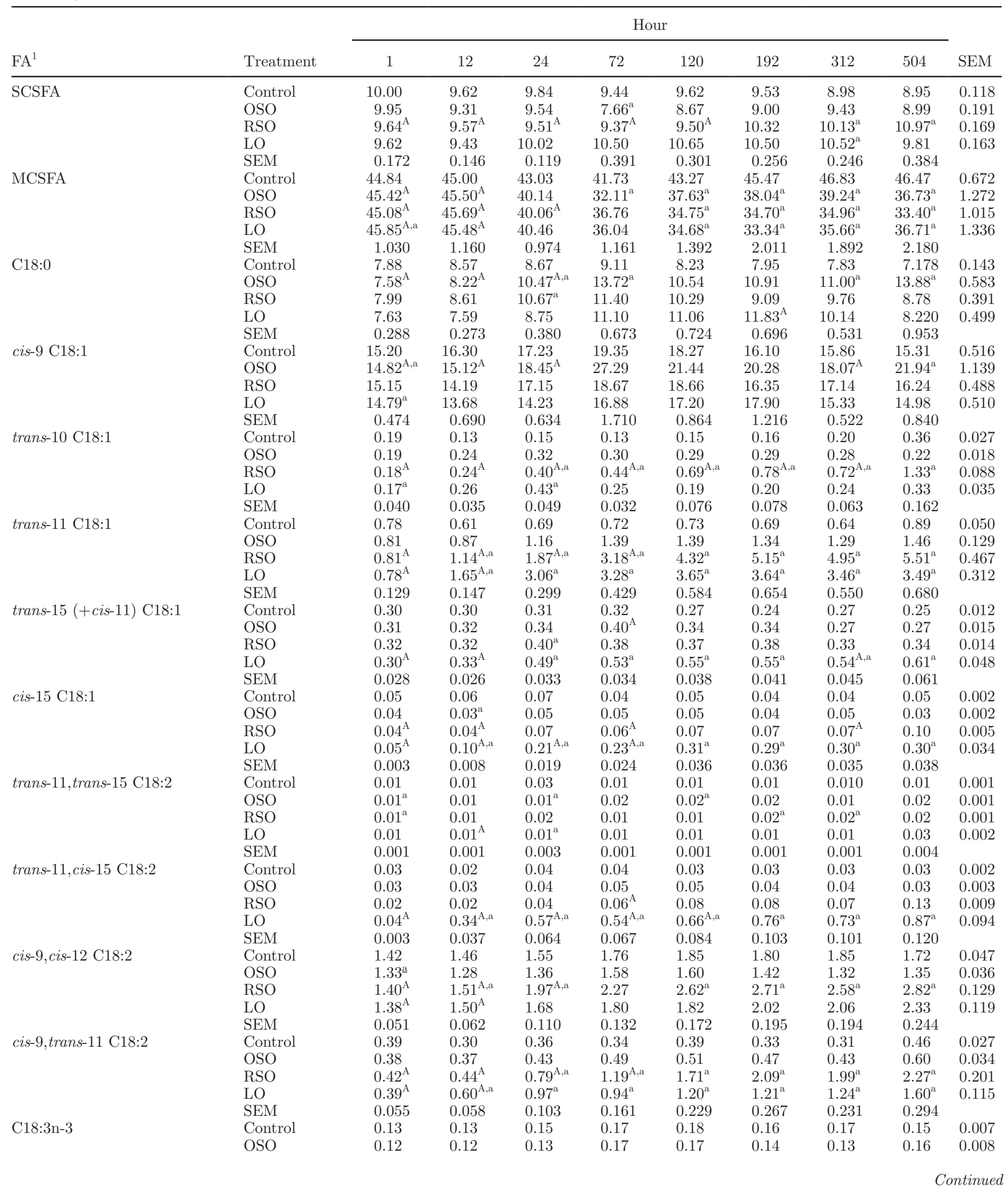


Table 2 (Continued). Fatty acid content (g/100 g of FA methyl esters) at different sampling times in milk fat from goats fed control (basal diet with no added oil) or oil-supplemented diets

\begin{tabular}{|c|c|c|c|c|c|c|c|c|c|c|}
\hline $\mathrm{FA}^{1}$ & Treatment & \multicolumn{8}{|c|}{ Hour } & SEM \\
\hline \multirow{5}{*}{ cis-9,trans-11,trans-15 C18:3 } & RSO & 0.15 & 0.12 & 0.18 & 0.15 & 0.17 & 0.14 & $0.14^{\mathrm{A}}$ & 0.18 & 0.006 \\
\hline & SEM & 0.007 & 0.025 & 0.041 & 0.044 & 0.051 & 0.062 & 0.078 & 0.112 & \\
\hline & Control & $\operatorname{Tr}^{2}$ & $\operatorname{Tr}$ & $\operatorname{Tr}$ & $\operatorname{Tr}$ & $\operatorname{Tr}$ & $\operatorname{Tr}$ & $\operatorname{Tr}$ & $\operatorname{Tr}$ & 0.000 \\
\hline & OSO & $\operatorname{Tr}$ & $\operatorname{Tr}$ & $\operatorname{Tr}$ & $\operatorname{Tr}$ & $\operatorname{Tr}$ & $\operatorname{Tr}$ & $\operatorname{Tr}$ & $\operatorname{Tr}$ & 0.000 \\
\hline & SEM & 0.000 & 0.001 & 0.002 & 0.003 & 0.004 & 0.005 & 0.004 & 0.006 & \\
\hline \multirow[t]{5}{*}{ cis-9,trans-11,cis-15 C18:3 } & Control & 0.05 & $0.06^{\mathrm{A}}$ & $0.05^{\mathrm{A}}$ & $0.05^{\mathrm{A}}$ & 0.04 & $0.04^{\mathrm{A}}$ & 0.04 & 0.03 & 0.005 \\
\hline & OSO & 0.04 & 0.05 & 0.05 & 0.04 & 0.04 & 0.04 & 0.03 & 0.03 & 0.002 \\
\hline & RSO & 0.04 & 0.04 & 0.04 & 0.05 & 0.04 & 0.04 & 0.04 & 0.04 & 0.001 \\
\hline & $\mathrm{LO}$ & $0.03^{\mathrm{A}}$ & $0.07^{\mathrm{A}}$ & $0.09^{\mathrm{a}}$ & $0.09^{\mathrm{a}}$ & $0.09^{\mathrm{a}}$ & $0.11^{\mathrm{a}}$ & $0.14^{\mathrm{A}, \mathrm{a}}$ & $0.10^{\mathrm{a}}$ & 0.012 \\
\hline & SEM & 0.005 & 0.007 & 0.008 & 0.008 & 0.008 & 0.011 & 0.017 & 0.013 & \\
\hline
\end{tabular}

${ }^{\mathrm{A}}$ Within an FA, the means in a treatment with superscript "A" differ significantly $(P<0.05)$ from the 504 -h mean in the same treatment. ${ }^{a}$ Within an FA, the means in OSO, RSO, or LO (diets enriched with $48 \mathrm{~g} / \mathrm{d}$ of high oleic sunflower oil, regular sunflower oil, or linseed oil, respectively) treatments with superscript "a" differ significantly $(P<0.05)$ from the same mean in the control treatment.

${ }^{1}$ Short-chain SFA (SCSFA) = sum of C4:0, C6:0, and C8:0; medium-chain SFA (MCSFA) = sum of C12:0, C14:0, and C16:0.

${ }^{2} \mathrm{Tr}=$ concentrations below $0.01 \mathrm{~g} / 100 \mathrm{~g}$ of FA methyl esters.

These results indicate that the response of trans-11 C18:1 and cis-9,trans-11 C18:2 to diets supplemented with LO was faster but weaker than with RSO. Bernard et al. (2009) also found higher trans-11 C18:1 and cis-9,trans-11 C18:2 contents in goat milk fat when the basal diet was supplemented with sunflower oil instead of LO.

In the LO treatment, the contents of trans-11 C18:1 and cis-9,trans-11 C18:2 were not different from their respective 504-h values at $24 \mathrm{~h}$ (i.e., a plateau was reached up to the final sampling time), but in the RSO treatment, the plateau started later (120 h; Figure 1 and Table 2). Work in vitro by Jouany et al. (2007) with a mixed rumen microbial population indicated that $\mathrm{BH}$ of $\mathrm{C} 18: 3 \mathrm{n}-3$ was faster, reaching a maximum at $5 \mathrm{~h}$, whereas, in the case of cis-9,cis-12 C18:2, BH was still important between 5 and $24 \mathrm{~h}$. According to Jouany et al. (2007), the reason for the delayed BH of cis-9,cis-12 C18:2 could be due to the preferential uptake of 18:3n-3 by bacteria, or differences in microbial isomerase or saturase affinity between the $2 \mathrm{FA}$. Work with dairy goats by Chilliard et al. (2005) indicated that the cis-9,trans-11 C18:2 increase in milk fat of goats fed sunflower and linseed oils, which was observed at $7 \mathrm{~d}$, lasted up to the end of the experiment $(35 \mathrm{~d})$. Luna et al. (2008), also working with goats supplemented with whole linseed and sunflower oil, found that the maximum values in milk fat of trans-11 $\mathrm{C} 18: 1$ and cis9,trans-11 C18:2 were at $15 \mathrm{~d}$. Unfortunately, no data was reported for the week before supplementation in those experiments.

\section{Comparing Oil Treatments with the Control}

Comparison of FA contents from oil treatments with the corresponding values in the control treatment at the different sampling times are shown in Table 2. Of the 45 possible comparisons (i.e., 15 selected FA and 3 oil treatments), 28 (7, 9, and 12 in OSO, RSO, and LO treatments, respectively) had significantly different values from the corresponding values in the control at 1 or more sampling times. Out of these 28 comparisons, 16 showed differences of the same algebraic sign (positive or negative) to their corresponding 312- and 504-h values in the control treatment. All 3 oils lowered the MCSFA content in milk fat, whereas increases in trans-11 C18:1 and cis-9,trans-11 C18:2 content were common to RSO and LO treatments. Specific for each oil were the increases in C18:0 in the OSO treatment; SCSFA, trans-10 C18:1, and cis-9,cis-12 C18:2 in the RSO treatment; and trans-15(+cis-11) C18:1, cis-15 C18:1, trans-11,cis-15 C18:2, C18:3n-3, and cis-9,trans11,cis-15 C18:3 in the LO treatment.

Some of the changes observed when comparing oilsupplemented diets with the control diet at 312 and $504 \mathrm{~h}$ were also detected at shorter time periods. For example, the MCSFA content in milk fat of goats fed oil-supplemented diets had the same response observed at 312 and $504 \mathrm{~h}$ and as soon as $120 \mathrm{~h}$; that response was already present at $72 \mathrm{~h}$ in the OSO treatment. Other FA responded faster: cis-9,trans-11 C18:2 and trans-10 C18:1 contents in milk fat of RSO-supplemented goats and cis-9,trans-11,cis-15 C18:3 in milk fat of LO-supplemented goats were already different from the 

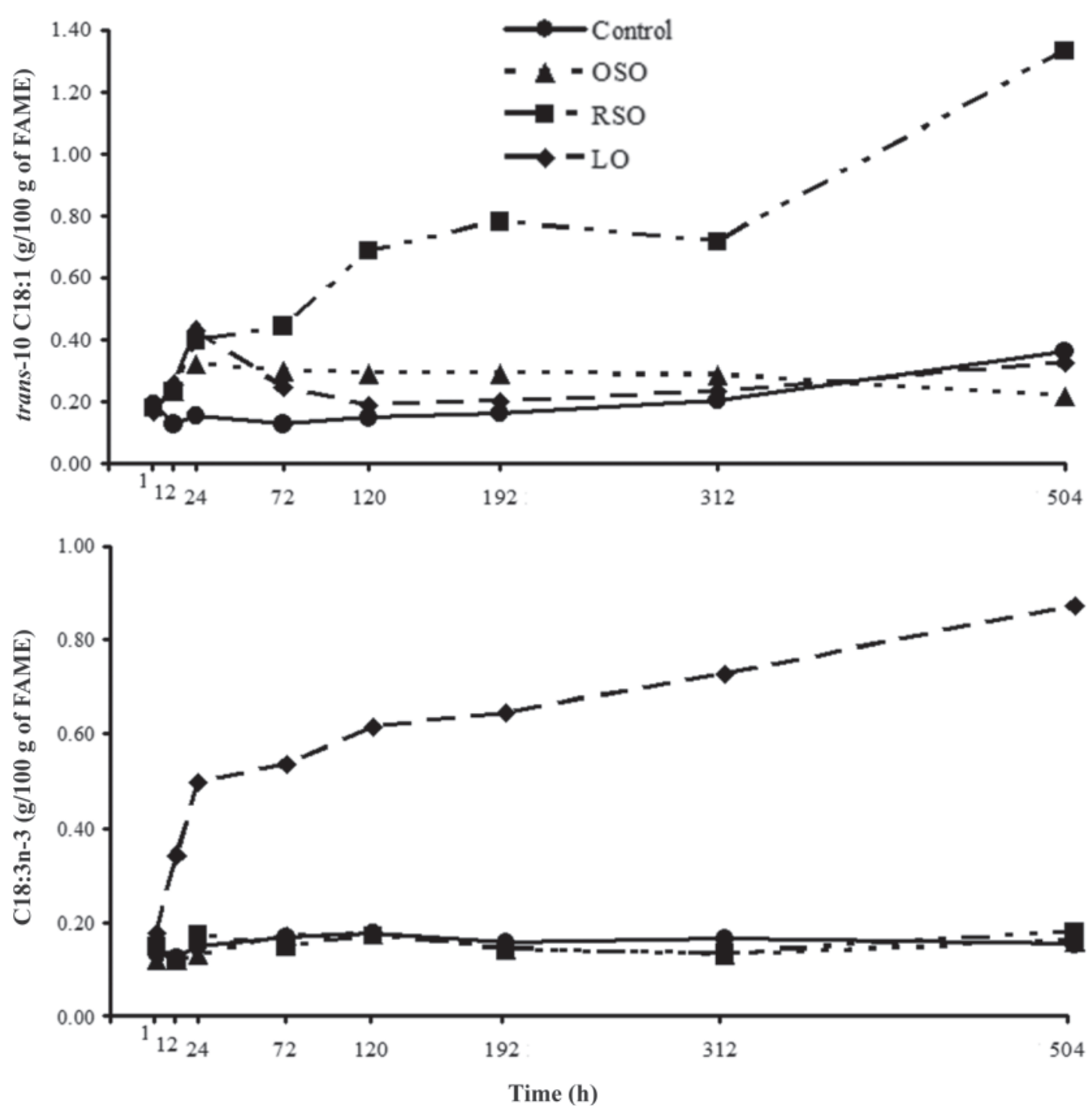

Figure 2. Time-dependent changes of trans-10 C18:1 and C18:3n-3 in milk fat of goats fed an unsupplemented diet (control) or diets enriched with high oleic sunflower oil (OSO), regular sunflower oil (RSO), or linseed oil (LO). FAME = FA methyl esters.

control at $24 \mathrm{~h}$. Differences observed with the control treatment appeared at $12 \mathrm{~h}$ for trans-11 C18:1 in both RSO and LO treatments, for cis-9,cis-12 C18:2 in the RSO treatment, and for cis-15 C18:1, cis-9,trans-11 C18:2, and trans-11,cis-15 C18:2 in the LO treatment. Furthermore, only $1 \mathrm{~h}$ was necessary to obtain a significant increase in C18:3n-3 in the LO treatment. All the responses mentioned in the paragraph above were consistent in that the differences with control values were statistically significant at all sampling times after the earlier one observed. In contrast, other FA comparisons failed to give a continuous significant response after the first difference with the control was seen (Table 2).

The fact that the milk FA profile can be changed shortly after dietary lipid introduction had already been observed by Scott et al. (1971) and Gulati et al.
(1997). Those authors introduced fat into diets and observed clear changes in milk fat FA composition at the first milk sampling at $36 \mathrm{~h}$ in cows and $96 \mathrm{~h}$ in goats. It can be argued that those authors used protected oils or oilseeds, but the relevant fact is the short time required to obtain clear results. This is not surprising, in view of the results obtained by Li et al. (2009). Those authors observed increased cis-9,trans-11 C18:2 in the rumen fluid of dairy goats fed twice daily a diet supplemented with $4 \%$ soybean oil at 1, 2, and $6 \mathrm{~h}$ after the morning feeding.

The observed evolution of FA contents determined in the present work could not be necessarily identical for other types of diets (i.e., when lipid supplementation is accompanied by other changes, such as the dietary forage-to-concentrate ratio). However, a shortening of 
the period for assessing changes in milk fat following the introduction of dietary lipid supplements may be possible. Although the data suggest that longer periods after lipid supplementation would be required for an accurate assessment of some FA (mainly trans-10 C18:1), a stabile milk fat general profile could be achieved at shorter times than $21 \mathrm{~d}$.

\section{CONCLUSIONS}

In summary, compared with their respective 504-h values, most FA responding to the addition of any of the 3 plant oils used in this study reached a value that did not differ greatly between 24 and 192 h (i.e., between 1 and $8 \mathrm{~d}$ ). That means that for most of the FA studied, the time from introducing the plant oil into the diet up to having a milk fat response not different from that obtained at $21 \mathrm{~d}$ was $8 \mathrm{~d}$. Results obtained under the conditions described in this paper show that typical changes in milk fat FA content by separately adding 3 different plant oils (OSO, RSO, or LO) to the diet of dairy goats, compared with a control diet without added fat, can be obtained as soon as $13 \mathrm{~d}$ after introducing the oils into the diet for most FA (earlier for many of them), although the maximum attainable values for some FA may require a longer time.

\section{ACKNOWLEDGMENTS}

This work was financed by the Ministerio de Ciencia e Innovación (MICINN, Madrid, Spain; AGL2008-04805 and the Consolider Ingenio 2010 Programme; FUN-CFOOD CSD2007-063) and the Comunidad Autónoma de Madrid (Spain; 2009-AGR-1469). The authors thank A. Jiménez Secilla and B. Garfia Catalán of Laboratorio Agropecuario Regional of Córdoba (Spain) for their help with milk composition analysis. We are also grateful to I. Andújar-Ramírez (Universidad de Córdoba, Córdoba, Spain) and M. V. Rodríguez-Pino [Consejo Superior de Investigaciones Cientificas (CSIC), Madrid, Spain] for their technical assistance in processing milk samples and J. J. Pérez-Hernández (Universidad de Córdoba) for his assistance in animal feeding and management.

\section{REFERENCES}

Bernard, L., K. J. Shingfield, J. Rouel, A. Ferlay, and Y. Chilliard. 2009. Effect of plant oils in the diet on performance and milk fatty acid composition in goats fed diets based on grass hay or maize silage. Br. J. Nutr. 101:213-224.

Chilliard, Y., J. Rouel, P. Guillouet, K. Raynal-Ljutovac, L. Leloutre, and A. Ferlay. 2005. Kinetics of responses of goat milk fatty acids to dietary forage:concentrate ratio and/or high doses of sunflower or linseed oil, or extruded mixture of seeds. Page 268 in Book of Abstracts, 56th Annual Meeting of European Association for Animal Production. Wageningen Academic Publishers, Uppsala, Sweden.
Dhiman, T. R., L. D. Satter, M. W. Pariza, M. P. Galli, K. Albright, and M. X. Tolosa. 2000. Conjugated linoleic acid (CLA) content of milk from cows offered diets rich in linoleic and linolenic acid. J. Dairy Sci. 83:1016-1027.

Drackley, J. K., T. R. Overton, G. Ortiz-Gonzalez, A. D. Beaulieu, D. M. Barbano, J. M. Lynch, and E. G. Perkins. 2007. Responses to increasing amounts of high-oleic sunflower fatty acids infused into the abomasum of lactating dairy cows. J. Dairy Sci. 90:51655175 .

Fievez, V., B. Vlaeminck, T. Jenkins, F. Enjalbert, and M. Doreau. 2007. Assessing rumen biohydrogenation and its manipulation in vivo, in vitro and in situ. Eur. J. Lipid Sci. Technol. 109:740-756.

Gómez-Cortés, P., M. A. de la Fuente, P. G. Toral, P. Frutos, M. Juárez, and G. Hervás. 2011. Effects of different forage:concentrate ratio in dairy ewe diets supplemented with sunflower oil on animal performance and milk fatty acid profile. J. Dairy Sci. 94:45784588.

Gómez-Cortés, P., P. Frutos, A. R. Mantecón, M. Juárez, M. A. de la Fuente, and G. Hervás. 2008a. Milk production, conjugated linoleic acid content, and in vitro ruminal fermentation in response to high levels of soybean oil in dairy ewe diet. J. Dairy Sci. 91:1560-1569.

Gómez-Cortés, P., P. Frutos, A. R. Mantecón, M. Juárez, M. A. de la Fuente, and G. Hervás. 2008b. Addition of olive oil to dairy ewe diets: Effect on milk fatty acid profile and animal performance. J. Dairy Sci. 91:3119-3127.

Gómez-Cortés, P., C. Tyburczy, J. T. Brenna, M. Juárez, and M. A. de la Fuente. 2009. Characterization of cis-9 trans-11 trans-15 C18:3 in milk fat by GC and covalent adduct chemical ionization tandem MS. J. Lipid Res. 50:2412-2420.

Gulati, S. K., E. B. Byers, Y. G. Byers, J. R. Ashes, and T. W. Scott. 1997. Effect of feeding different fat supplements on the fatty acid composition of goat milk. Anim. Feed Sci. Technol. 66:159-164.

Harvatine, K. J., and M. S. Allen. 2006. Fat supplements affect fractional rates of ruminal fatty acid biohydrogenation and passage in dairy cows. J. Nutr. 136:677-685.

Hervás, G., P. Luna, A. R. Mantecón, N. Castañares, M. A. de la Fuente, M. Juárez, and P. Frutos. 2008. Effect of diet supplementation with sunflower oil in milk production, fatty acid profile and ruminal fermentation in lactating dairy ewes. J. Dairy Res. 75:399-405.

INRA (Institut National de la Recherche Agronomique). 2002. Tables de composition et de valeur nutritive des matières premières destinées aux animaux d'élevage. INRA, Paris, France.

ISO-IDF (International Organization for Standardization-International Dairy Federation). 2002a. Milk fat-Preparation of fatty acid methyl esters. International Standard ISO 15884:2002 (IDF 182: 2002). IDF, Brussels, Belgium.

ISO-IDF (International Organization for Standardization-International Dairy Federation). 2002b. Milk fat-Determination of the fatty acid composition by gas-liquid chromatography. International Standard ISO 15885:2002 [IDF 184:2002 (E)].

Jouany, J. P., B. Lassalas, M. Doreau, and F. Glasser. 2007. Dynamic features of the rumen metabolism of linoleic acid, linolenic acid and linseed oil measured in vitro. Lipids 42:351-360.

Khas-Erdene, J. Q. Wang, D. P. Bu, L. Wang, J. K. Drackley, Q. S. Liu, G. Yang, H. Y. Wei, and L. Y. Zhou. 2010. Responses to increasing amounts of free $\alpha$-linolenic acid infused into the duodenum of lactating dairy cows. J. Dairy Sci. 93:1677-1684.

Li, X. Z., C. G. Yang, R. J. Long, G. L. Jing, J. Shine Khuu, B. J. Ji, S. H. Choi, H. G. Lee, and M. K. Song. 2009. Conjugated linoleic acid in rumen fluid and milk fat, and methane emission of lactating goats fed a soybean oil-based diet supplemented with sodium bicarbonate and monensin. Asian-australas. J. Anim. Sci. 22:1521-1530.

Littell, R. C., G. A. Milliken, W. W. Stroup, R. D. Wolfinger, and O. Schabenberger. 2006. SAS for Mixed Models. 2nd ed. SAS Institute Inc., Cary NC.

Luna, P., A. Bach, M. Juárez, and M. A. de la Fuente. 2008. Effect of a diet enriched in whole linseed and sunflower oil on goat milk fatty acid composition and conjugated linoleic acid isomer profile. J. Dairy Sci. 91:20-28. 
Luna, P., J. Fontecha, M. Juárez, and M. A. de la Fuente. 2005. Changes in the milk and cheese fat composition of ewes fed commercial supplements containing linseed with special reference to the CLA content and isomer composition. Lipids 40:445-454.

Martínez Marín, A. L., P. Gómez-Cortés, G. Gómez Castro, M. Juárez, L. Pérez Alba, M. Pérez Hernández, and M. A. de la Fuente. 2012. Effects of feeding increasing dietary levels of high oleic or regular sunflower or linseed oil on fatty acid profile of goat milk. J. Dairy Sci. 95:1942-1955.

Moate, P. J., W. Chalupa, T. C. Jenkins, and R. C. Boston. 2004. A model to describe ruminal metabolism and intestinal absorption of long chain fatty acids. Anim. Feed Sci. Technol. 112:79-105.

Mosley, E. E., G. L. Powell, M. B. Riley, and T. C. Jenkins. 2002. Microbial biohydrogenation of oleic acid to trans isomers in vitro. J. Lipid Res. 43:290-296.

NRC. 2007. Nutrient Requirements of Small Ruminants. National Academy Press, Washington DC.
Roy, A., A. Ferlay, K. J. Shingfield, and Y. Chilliard. 2006. Examination of the persistency of milk fatty acid composition responses to plant oils in cows given different basal diets, with particular emphasis on trans-C18:1 fatty acids and isomers of conjugated linoleic acid. Anim. Sci. 82:479-492.

SAS Institute. 2004. SAS/STAT 9.1 User's Guide. SAS Institute Inc., Cary, NC.

Scott, T. W., L. J. Cook, and S. C. Mills. 1971. Protection of dietary polyunsaturated fatty acids against microbial hydrogenation in ruminants. J. Am. Oil Chem. Soc. 48:358-364.

Shingfield, K. J., C. K. Reynolds, G. Hervás, J. M. Griinari, A. S. Grandison, and D. E. Beever. 2006. Examination of the persistency of milk fatty acid composition responses to fish oil and sunflower oil in the diet of dairy cows. J. Dairy Sci. 89:714-732. 